P. C. H. Martens, S. Tsuruta, and M. A. Weber, eds.

\title{
The Scaling of Solar Flare Hard X-ray Emission to Other Flaring Objects in the Universe
}

\author{
P. C. H. Martens \\ Department of Physics, Montana State University-Bozeman, \\ Bozeman, MT 59717-3840, U.S.A.
}

\begin{abstract}
Fletcher \& Martens have successfully modeled solar hard Xray sources observed at the top and footpoints of flaring magnetic loops with a Fokker-Planck type particle transport code. I show here that there are invariances in the Fokker-Planck equations that make these results applicable to environments with vastly different physical parameters, such as hard X-ray flares in accretion disks in active galactic nuclei, and in RS CVn and ALGOL type binaries.
\end{abstract}

The hard and soft X-ray telescopes onboard the Japanese Yohkoh spacecraft have unambiguously established that solar flare hard X-ray (HXR) emission originates from the footpoints of magnetic loops, as well as from the tops of these same loops (Masuda et al. 1994).

Fletcher \& Martens (1998) model the HXR emission from the looptops and the footpoints, using the commonly observed cusped loop geometry with a current-sheet on top, and they find a looptop and two footpoint HXR sources akin to those observed by Yohkoh. The Fletcher \& Martens model relies on a numerical solution of the Fokker-Planck equation for the propagation of the electron beam injected at the looptop. In dimensionless units, their model equation reads

$$
\begin{aligned}
& \frac{\partial f}{\partial t}+\mu v \frac{\partial f}{\partial z}-\varepsilon \frac{\partial}{\partial E}\left(\frac{f}{v}\right)- \frac{v}{2} \frac{\partial}{\partial \mu}\left(\left(1-\mu^{2}\right) \frac{\mathrm{d} \ln \mathrm{B}}{\mathrm{dz}} f\right)- \\
&-\varepsilon \frac{\partial}{\partial \mu}\left(\left(1-\mu^{2}\right) \frac{\partial f}{\partial \mu}\right)=0
\end{aligned}
$$

where $f(z, t, \mu)$ is the particle distribution as a function of time $(t)$, position along a fieldline $(z)$, and pitch-angle $(\mu) ; B$ is the magnetic field strength, $E$ the particle energy, and $v$ the particle velocity. This dimensionless form is found by defining the units (subscript zero) $t_{0}=L / v_{0}, E_{0}=\frac{m_{e}}{2} v_{0}^{2}$, and $\varepsilon=\frac{\pi e^{2} \Lambda n_{e} L}{E_{0}^{2}}$. Here, $L$ is the loop length from top to footpoint, $\Lambda$ is the Coulomb-factor $(\approx 20)$, while $m_{e}, n_{e}$, and $e$ are the mass, density, and charge of the electrons.

The only parameter in this equation is $\varepsilon$ which equals the ratio of the electron crossing time and the isotropization time of the electron beam. Therefore, $\varepsilon \leq 1$ implies the beam reaches the footpoints of a flaring loop only partially attenuated, and $\varepsilon>1$ implies the beam will diffuse before reaching the footpoints. Typical values for the solar corona are $L=2 \times 10^{9} \mathrm{~cm}, n_{e}=4 \times 10^{9} \mathrm{~cm}^{-3}$, and $E_{0}=30 \mathrm{keV}$. Hence, $v_{0}=c / 3, \quad t_{0}=0.2 \mathrm{~s}$, and $\varepsilon=0.2$. 
The initial condition for the electron beam distribution function inferred from observations is a power law in energy, and a sharply peaked (beamed) function in pitch-angle, which is invariant for the choices of the dimensionality parameters that determine $\varepsilon$. Thus, with these initial conditions, the value of $\varepsilon$ completely determines the character of the solutions, and one can simply scale solutions from one part of parameter space to another for a given value of $\varepsilon$.

Let us examine two simple examples of scaling invariance. Consider two flaring loops, one five times longer than the other $\left(L_{n e w}=5 \times L_{\text {old }}\right)$, and keep the column depth, $n_{e} L$, and hence $\varepsilon$, constant. All solutions will be identical, except the new time unit, $t_{0}=\frac{L_{\text {new }}}{v_{0}}$, is five times larger, i.e., the HXR evolution is five times slower.

In the second example, I keep the electron density $n_{e}$ constant, and choose $L_{\text {new }}=4 \times L_{\text {old }}$ and $E_{0 \text { new }}=2 \times E_{0 \text { old }}$. Hence, $\varepsilon$ is preserved, and with the same power-law initial conditions, all solutions are identical in form, with the $30 \mathrm{keV}$ emission profile shifted to $60 \mathrm{keV}$, and the time unit $\left(t_{0}=L_{n e w} / v_{0}\right)$ a factor $2 \sqrt{2}$ times larger.

Typical parameters for magnetic loops in an accretion disk in an active galactic nucleus are $L \approx 3 \times 10^{7} \mathrm{~cm}$ and $n_{e} \approx 2 \times 10^{18} \mathrm{~cm}^{-3}$ (de Vries \& Kuijpers 1992). Therefrom, I find that $\varepsilon \approx 5 \times 10^{5}$, so that the flaring loop is completely optically thick for $30 \mathrm{keV}$ electrons. One would observe a looptop source, but no footpoints, in the keV range. The energy spectrum is that of a thick target source, and typical timescales would be much shorter than in the solar case (since the looplength is much less). A looptop source originating from magnetic trapping, plus footpoint sources, can only be expected for $60 \mathrm{MeV}$ electrons (and $\mathrm{GeV}$ range protons), but the amount of particles generated at these energies is usually much lower than at $\mathrm{keV}$ ranges.

Typical members of the families of RSCVn and Algol type binaries are Algol itself and $\sigma^{2}$ CrB. From Exosat observations, van den Oord, Mewe, \& Brinkman (1988) find that Algol has magnetic loops with densities of the order of $3 \times 10^{11} \mathrm{~cm}^{-3}$, and looplength $7.8 \times 10^{10} \mathrm{~cm}$, while $\sigma^{2} \mathrm{CrB}$ has respectively 3 $\times 10^{11} \mathrm{~cm}^{-3}$ and $4.5 \times 10^{10} \mathrm{~cm}$. Their flare X-ray luminosities are respectively one and two orders of magnitude larger than that of the Sun. For both binary systems, $\varepsilon \gg 1$ in the keV energy range, and hence once more, one would observe a HXR looptop source but no footpoints. HXR footpoints would emerge above about $1.5 \mathrm{MeV}$.

We conclude that the Fletcher \& Martens (1998) simulations can be used without much adaptation to generate HXR energy spectra and evolution curves for a large variety of flaring astrophysical objects.

\section{References}

de Vries, M., \& Kuijpers, J. 1992, A\&A, 266, 77

Fletcher, L., \& Martens, P. C. H. 1998, ApJ, 505, 418

Masuda, S., Kosugi, T., Hara, H., Tsuneta, S., \& Ogawara, Y. 1994, Nature, 371,495

van den Oord, G. H. J., Mewe, R., \& Brinkman, A. C. 1988, A\&A, 205, 181 